\title{
Conditional Unitary Transformation on biphotons
}

\author{
G. Brida ${ }^{1}$, M.V. Chekhova ${ }^{2}$, M. Genovese ${ }^{1}$, M. Gramegna ${ }^{1}$, L.A. Krivitsky ${ }^{2}$, S.P.Kulik ${ }^{2}$ \\ 1 Istituto Elettrotecnico Nazionale Galileo Ferraris, \\ Strada delle Cacce 91, 10135 Torino, Italy \\ 2 Physics Department, M. V. Lomonosov Moscow State University, 119992 Moscow, Russia.
}

\begin{abstract}
A conditional unitary transformation ( $90^{\circ}$ polarization rotation) is performed at singlephoton level. The transformation is realized by rotating polarization for one of the photons of a polarization-entangled biphoton state (signal photon) by means of a Pockel cell triggered by the detection of the other (idler) photon after polarization selection. As a result, the state of the signal photon is losslessly changed from being completely unpolarized to being partially polarized, so that the final polarization degree is given by the idler detector quantum efficiency. This experiment can be used for developing a novel method of absolute quantum efficiency calibration.
\end{abstract}

PACS 03.67.-a; 03.65.Ta

The possibility of performing a unitary transformation on a qubit conditionally on the result of the measurement on another one is a fundamental tool for quantum information [1]. For example, in teleportation protocols [2], teleportation is performed by Bob doing an opportune unitary transformation on his sub-part of an entangled system after having received classical information on the result of the Bell measurement performed by Alice on her sub-part of the entangled system and the unknown state to be teleported. Similarly, in entanglement swapping protocols a joint measurement performed on two members of two distinct entangled pairs allows, by means of a conditional unitary transformation, to create a desired entangled state between the two surviving members. In most of experiments realized up to now with single photons both for teleportation [3] and swapping [4], this part of the protocol was not accomplished, and only correlation measurements with a fixed polarization 
selection were obtained. The only exception is [5], where a Pockel cell was used for conditional transformations in an active teleportation protocol. Conditional transformations were also performed in experiments where one of the photons of an entangled pair triggered polarization rotation on the other one, to increase the efficiency of the parity check [6] and to produce signal photons on 'pseudodemand', i.e., in a given time interval after the trigger detection event [7]. Single-photon light was observed through the anti-bunching effect in [9] as well, following a proposal of [8] where it was suggested to prepare pure single-photon state by gating (using a shutter) the signal photon of an entangled pair as soon as the idler photon is detected. Finally, for the sake of completeness, it can still be acknowledged that, somewhat later, it was proposed to produce sub-Poissonian light by means of conditional transformations on twin beams [10]. Conditional preparation of sub-Poissonian light from twin beams was carried out in experiments using active control (feedforward) [11] and passive measurement technique [12].

The idea of the present work is to perform conditional unitary transformations on the polarization state of a photon belonging to a polarization-entangled pair. This experiment is the first demonstration of 'purifying' the polarization state of a photon that is initially prepared in a mixed polarization state (initial degree of polarization is equal to zero). As a result of transformations, the degree of polarization increases; its final value is given by the quantum efficiency of the 'trigger' detector. Due to this fact, one can notice another important advantage of our experiment: it can be used for the absolute calibration of photodetectors (absolute measurement of quantum efficiency).

In our experiment, biphotons are generated via spontaneous parametric down conversion (SPDC) by pumping a BBO crystal $(5 \times 5 \times 5 \mathrm{~mm})$ with an argon laser radiating at $351 \mathrm{~nm}$. The crystal is cut for type-II non-collinear frequency-degenerate phase matching providing generation of polarization-wavevector entangled state [13]. In this regime, signal and idler photons are emitted in different directions and each of them is not polarized; at the same time, their polarization states are always orthogonal. The state at the output of the crystal has the form

$$
|\psi\rangle=\frac{|H\rangle|V\rangle+\mathrm{e}^{\mathrm{i} \phi}|\mathrm{V}\rangle|\mathrm{H}\rangle}{\sqrt{2}},
$$

where $H, V$ denote horizontal and vertical polarization, respectively, and the first and the second positions correspond to the two spatial modes (two directions). The phase $\phi$ can be 
varied by small tilts of the crystal and by additionally introduced optic elements.

The state (1) is a pure entangled state. Strictly speaking, in order to obtain this state, it is necessary that the group delay between vertically and horizontally polarized photons is compensated after the crystal [14]. As a compensator, one can use a BBO crystal of length twice less than for the one where photon pairs are generated, or any other crystal of equivalent length. Without this compensation, the phase $\phi$ in Eq. (1) has classical random fluctuations or, in other words, the state produced at the output is a mixed entangled state, described by the density matrix

$$
\rho=\frac{|H V\rangle\langle H V|+| V H\rangle\langle V H|}{2} .
$$

In our experiment, no compensator was used, and hence, a mixed entangled state (2) was generated. Note that each of the photons forming the entangled pair is in a mixed polarization state, no matter whether the two-photon state is pure (Eq.(1)) or mixed (Eq.(2)). The effect of 'purification' observed in the present work for the polarization state of the signal photon does not require the purity of the two-photon state. Some additional features should be observed in the case where the two-photon state is pure, but this is not the subject of the present paper and will be considered elsewhere.

After the BBO crystal, the pump was absorbed by a beam trap. One of the correlated photons (the idler photon) was sent to a single-photon counter (Perkin-Elmer SPCM-AQ) preceded by a red-glass cutoff filter and a pinhole (realizing a rough spectral and spatial selection) and then to a polarizer cube selecting vertically polarized photons. The output of this detector was sent to a counter and, as a trigger, to a pulse generator used to control a KDP Pockel cell placed on the path of the conjugated (signal) photon. The Pockel cell was operating as a halfwave plate oriented at $45^{\circ}$ to the vertical axis (thus providing polarization rotation by $90^{\circ}$ ) if supplied by the $5.2 \mathrm{kV}$ peak voltage from the pulse generator. The highvoltage pulse had a sharp front, not longer than 2 ns, a flat part of duration 100 ns, and a 'tail' $2 \mu \mathrm{s}$ long. To ensure that the flat part of the high-voltage pulse arrived at the Pockel cell simultaneously with the signal photon, signal photons were delayed by means of $50 \mathrm{~m}$ of polarization-maintaining (PM) fibre. For additional adjustment, a variable delay between the idler detector output pulse and the high-voltage pulse could be introduced electronically. This way, each detection of an idler photon with vertical polarisation led to a unitary operation (polarisation rotation by $90^{\circ}$ ) on the corresponding signal photon. Then, 
after an interference filter with $4 \mathrm{~nm}$ FWHM centered at $702 \mathrm{~nm}$ and a Glan-Thompson polarizer, the signal photon was detected by a single-photon counter (Perkin-Elmer SPCMAQ).

When the Pockel cell is not active, the reduced polarization density matrix of the signal photon corresponds to a completely unpolarized case. In fact, the observed dependence of the signal beam intensity on the setting of the Glan-Thompson polarizer was flat (see Fig.2).

On the other hand, when the Pockel cell is active, the system realizes a unitary transformation (rotation by $90^{\circ}$ ) on the polarization of the signal photon conditioned to the registration of a vertically polarized idler photon. The polarization density matrix of the signal photon corresponds therefore to a perfectly vertically polarized state (if the idler detector has perfect quantum efficiency and the Pockel cell operates in 100\% cases): the initially perfectly mixed state of the signal photon $1 / 2(|V\rangle\langle V|+| H\rangle\langle H|)$ is purified to the state $|V\rangle\langle V|$. In a real situation, the quantum efficiency $\eta$ of the first detector is smaller than unity, which means that only a fraction of the incident photons will be observed and produce an effect on the Pockel cell. Thus, the final density matrix for the second photon is

$$
\rho=1 / 2((1+\eta)|V\rangle\langle V|+(1-\eta)| H\rangle\langle H|)
$$

It is important that the transformation performed on the signal photon, in principle, is lossless, since the only sources of losses are reflection and absorption in the optical elements, which can be made as small as possible.

This effect can be also described in terms of the Stokes parameters, which are initially $S_{0}=N, S_{1}=S_{2}=S_{3}=0$, where $N$ is the photon number, but become, as a result of transformation, $S_{0}=N, S_{1}=\eta N, S_{2}=S_{3}=0$. From the Stokes parameters, one can find the polarization degree, which is standardly defined as

$$
P=\sqrt{\frac{S_{1}^{2}+S_{2}^{2}+S_{3}^{2}}{S_{0}^{2}}} .
$$

We see that without the transformation, $P=0$ and in the presence of the transformation, $P=\eta$. Since the degree of polarization depends on $\eta$ this scheme could also be used for an absolute calibration of detectors (a detailed description of this technique will appear in Ref. [15]).

The results of our measurements are shown in Figs.2, 3. As expected, in the absence of the Pockel cell action, the measured flux on the signal detector does not depend on the 
polarization selection. On the other hand, when the cell is active the measured signal has a $(1+\eta \cos (2 \theta))$ behaviour as a function of the polarizer setting $\theta$, with a $30 \%$ visibility (Fig.2). Subtracting the noise background of the signal detector and correcting for Pockel cell system inefficiency, we discover that the visibility becomes $0.441 \pm 0.045$, which is close to the result $\eta=0.476 \pm 0.002$ obtained by means of a standard biphoton calibration scheme $[16,17]$. The dependence of coincidence counting rate on the position of the polarizer in the signal channel is shown in Fig.3. In contrast to the single-photon counting rate, this dependence should not be sensitive to the idler detector quantum efficiency. The effect of the Pockel cell manifests itself in the $90^{\circ}$ phase shift of the dependence. A small decrease in the visibility (from 95\% to 90\%) can be explained only by 'failures' of the Pockel cell caused by the effect of its dead time $(2 \mu \mathrm{s})$.

To demonstrate that the effect occurs only in cases where the polarization transformation on the signal photon is triggered by its conjugated idler photon, we studied the dependence of the effect on the trigger pulse delay $T$ introduced electronically. To obtain this dependence, the electronic delay was scanned from 0 to 200 ns while the polarizer in the signal channel was fixed in the positions $\theta=0^{\circ}$ and $\theta=90^{\circ}$ (Fig.4). The dependence shows that at zero electronic delay, the effect is observed but at delays larger than 100 ns, the effect disappears. To explain this dependence, we have to mention that the electronic part of the setup has a constant uncontrollable delay $T_{0}$ introduced by the pulse generator. At zero additional delay $T$, the effect is present because the delay $T_{f}$ introduced by the fibre (248 ns, according to our measurement) is small enough compared to the delay $T_{0}$ plus the duration of the high-voltage pulse. Disappearance of the effect at $T>100$ ns occurs because in this case $T+T_{0}$ starts to exceed the delay $T_{f}$ introduced by the fibre. By taking a longer fibre we could observe no effect at small $T$ and at larger $T$, the initial part of the dependence would show the 'tail' of the high-voltage pulse; however, this tail is more than one microsecond long and the PM fibre does not maintain polarization well enough at lengths larger than several hundreds of meters.

In conclusion, we have performed a conditional unitary transformation at single-photon level. The state of the signal photon, which was initially mixed, became partly purified due to this transformation. In terms of polarization properties, light became partly polarized (polarization degree became equal to the quantum efficiency of the trigger detector) while initially, it was completely non-polarized (polarization degree was zero). Note that the 
transformation is lossless with respect to the signal photon. It is worth mentioning that variation of the polarization degree, which we observe in our experiment, is impossible by means of only linear lossless optical methods [18]. However, in the present experiment, the transformation performed over the signal photon is essentially nonlinear since it is triggered by the detection of the idler photon entangled to this photon.

\section{Acknowledgments}

We acknowledge support of INTAS, grant \#01-2122. One of us (L.K.) acknowledges support from INTAS-YS fellowship grant (Num. 03-55-1971). The Turin group acknowledges the support of: MIUR (FIRB RBAU01L5AZ-002; Cofinanziamento 2001) and Regione Piemonte. The Moscow group acknowledges support of the Russian Foundation for Basic Research, grant\#02-02-16664, and the Russian program of scientific school support (\#166.2003.02).

[1] "Quantum Computation and Quantum Information", M. A. Nielsen and I.L. Chuang, ed. Cambridge Univ. Press, Cambridge (2000); "The Physics of Quantum Information", D. Bouwmeester et al., ed. Springer Verlag, Berlin (2000).

[2] C.H. Bennett et al., Phys. Rev. Lett. 70, 1895 (1993).

[3] D. Bouwmeester et al., Nature 390, 575 (1997); D. Boschi et al., Phys. Rev. Lett. 80 (98) 1121; Y.-H. Kim et al., Phys. Rev. Lett. 86, 1370 (2001).

[4] J.-W. Pan et al., Phys. Rev. Lett. 80, 3891 (1998); T. Jennewein et al., Phys. Rev. Lett. 88, $017903(2002)$.

[5] S.Giacomini et. al.,Phys. Rev. A 66, 030302(R) (2002).

[6] T.B.Pittman, B.C.Jacobs, and J.D.Franson, Phys. Rev. A 66, 052305 (2002).

[7] T.B.Pittman, B.C.Jacobs, and J.D.Franson, Phys. Rev. A 66, 042303 (2002).

[8] D.N.Klyshko, Sov.J. Quant. Electronics, 7, 591 (1977). 
[9] J.G.Rarity, P.R.Tapster, and E.Jakeman, Optics Communications 62, 201 (1987).

[10] K.Watanabe and Y.Yamamoto, Phys. Rev. A 38, 3556 (1988).

[11] J.Mertz, A.Heidmann, and C.Fabre, Phys. Rev. A 44, 3229 (1991).

[12] J.Laurat et. al., Phys. Rev. Lett. 91, 213601 (2003).

[13] P.G.Kwiat et. al., Phys. Rev. Lett. 75, 4337 (1995).

[14] Y.H.Shih and A.V.Sergienko, Phys. Lett. A 186, 29 (1994).

[15] G. Brida, M. Chekhova, M. Genovese, M. Gramegna, L. Krivitsky, S. Kulik, M.L. Rastello, work in progress.

[16] D.C. Burnham and D.L. Weinberg, Phys. Rev. Lett. 25, 84 (1970); D.N. Klyshko, Sov. J. Quant. Elect. 10, 1112 (1980); A.A. Malygin, A.N. Penin, A.V. Sergienko, JETP Lett. 33477 (1981).

[17] G. Brida, M. Genovese and C. Novero, Jour. Mod. Opt. 47 (2000) 2099 and refs. therein.

[18] D.N.Klyshko, JETP 84 (6), 1065 (1997).

[19] After subtracting the noise counts we obtain the visibility of $44 \%$. 


\section{Figure Captions}

Fig.1. The experimental set-up. An argon laser generating at $351 \mathrm{~nm}$ pumps a type-II BBO crystal cut for frequency-degenerate non-collinear polarization-entangled phase matching. One of the correlated photons, after a spatial selection by means of an aperture A, a spectral selection by means of a red-glass cutoff filter RG, and a polarization selection by means of a polarizing cube PBS, is addressed to the photon counter D1, which drives, through a fast high-voltage switch S, a Pockel cell PC placed in the optical path of the other photon. The delay between a photocount of D1 and the corresponding high-voltage pulse on the Pockel cell can be varied electronically. The second photon of an entangled pair is retarded, before the Pockel cell, by means of a $50 \mathrm{~m}$ PM fibre F. This realizes the conditioned unitary operation. The second photon is registered by photon counter D2 preceded by a Glan prism G and an interference filter IF. The measurement includes registration of the D2 single-photon counting rate and the rate of coincidences between D1 and D2.

Fig.2. Counting rate of detector D2 as a function of the angle of the polarizer preceding

it. When the Pockel cell is not activated no dependence on the polarizer angle appears (squares). When a $90^{\circ}$ rotation of polarization is realized by the Pockel cell conditioned to the measurement of a vertically polarized photon in the conjugated branch, the data (triangles) show a clear dependence on the polarizer setting corresponding to a vertically polarized state, with a $30 \%$ visibility [19].

Fig.3. Coincidences between D1 and D2 as a function of the polarizer orientation. The data when the Pockel cell is activated show a $90 \%$ visibility. The reduction of visibility can be attributed to a non-perfect efficiency of the Pockel cell apparatus.

Fig.4. Dependence of the D2 counting rate at $\theta=0^{\circ}$ (Horizontally polarized photons, triangles) and $\theta=90^{\circ}$ (Vertically polarized photons, squares) on the delay $T$ introduced electronically between the trigger pulses from D1 detector and the corresponding highvoltage pulses driving the Pockel's cell. The effect of counting rate decreasing for horizontally polarized photons is observed for $T<100 \mathrm{~ns}$ and not observed for $T>100 \mathrm{~ns}$. 